\title{
REVIEW OF ELECTROCARDIOGRAM (ECG) FINDINGS IN PATIENTS WITH RESTRICTIVE EATING DISORDERS ATTENDING A REGIONAL CHILD AND ADOLESCENT EATING DISORDER SERVICE IN 2010
}

\author{
N. Barber ${ }^{1}$, S. Chapman ${ }^{2}$ \\ ${ }^{1}$ Paediatrics, Kings College Hospital NHS Foundation Trust, ${ }^{2}$ Child and Adolescent Eating Disorders \\ Service, Michael Rutter Centre, South London and the Maudsley NHS Foundation Trust, London, UK
}

Background/aims: Prolonged corrected QT interval (QTc) has been widely reported in patients with restrictive eating disorders, and is known to be associated with significant morbidity and mortality. We sought to identify the incidence of prolonged QTc and cardiac morbidity in all patients with restrictive eating disorders referred to a tertiary child and adolescent mental health eating disorder service in 2010.

Method: The notes of 101 patients referred in 2010 diagnosed with restrictive eating disorders were reviewed. All ECGs were were analysed for rate, rhythm, QTc (using Bazett's formula) and QTc dispersion. Demographic and anthropometric data, contemporaneous electrolyte values as well as information on cardiac symptomatology and medication history were also collected.

Results: The ECGs of 34 patients (33 female, mean age $15.1 \pm 2$ years, mean \%BMI $79.8 \pm 11.3 \%$ ) were analysed. Bradycardia was found in 22 cases. There was no correlation between age, weight or \%BMI with heart rate. Prolonged QTc was not identified in any patient, though QTc dispersion was inversely correlated to $\%$ BMI $(r=-0.32, p=0.039)$ and weight $(r=-0.339, p=0.031)$. Syncope was documented in one case.

Conclusions: ECG abnormalities occur commonly in young people with eating disorders. While we identified no cases of prolonged QTc, QTc dispersion was observed to be greater at lower weights. Close clinical and ECG surveillance is therefore important. 